\title{
The Effect of Sterile Acellular Dermal Matrix Use on Complication Rates in Implant-Based Immediate Breast Reconstructions
}

\author{
Jun Ho Lee, Youngsoo Park, Kyoung Wook Choi, Kyu-Jin Chung, Tae Gon Kim, Yong-Ha Kim \\ Department of Plastic and Reconstructive Surgery, Yeungnam University College of Medicine, Daegu, Korea
}

Background The use of acellular dermal matrix (ADM) in implant-based immediate breast reconstruction has been increasing. The current ADMs available for breast reconstruction are offered as aseptic or sterile. No published studies have compared aseptic and sterile ADM in implant-based immediate breast reconstruction. The authors performed a retrospective study to evaluate the outcomes of aseptic versus sterile ADM in implant-based immediate breast reconstruction.

Methods Implant-based immediate breast reconstructions with ADM conducted between April 2013 and January 2016 were included. The patients were divided into 2 groups: the aseptic ADM (AlloDerm) group and the sterile ADM (MegaDerm) group. Archived records were reviewed for demographic data and postoperative complication types and frequencies. The complications included were infection, flap necrosis, capsular contracture, seroma, hematoma, and explantation for any cause.

Results Twenty patients were reconstructed with aseptic ADM, and 68 patients with sterile ADM. Rates of infection ( $15.0 \%$ vs. $10.3 \%)$, flap necrosis ( $5.0 \%$ vs. $7.4 \%)$, capsular contracture $(20.0 \%$ vs. $14.7 \%)$, seroma ( $10.0 \%$ vs. $14.7 \%)$, hematoma ( $0 \%$ vs. $1.5 \%)$, and explantation $(10.0 \%$ vs. $8.8 \%)$ were not significantly different in the 2 groups.

Conclusions Sterile ADM did not provide better results regarding infectious complications than aseptic ADM in implant-based immediate breast reconstruction.

Keywords Breast implants / Acellular dermis / Mammaplasty
Correspondence: Jun Ho Lee Department of Plastic and Reconstructive Surgery, Yeungnam University College of Medicine, 170 Hyeonchung-ro, Nam-gu, Daegu 42415, Korea

Tel: +82-53-620-3482

Fax: +82-53-626-0705

E-mail: junojunho@gmail.com

This article was supported by Yeungnam University grants in 2014 .

This article was presented at Aesthetic Plastic Surgery 2014 on April 26, 2014 in Seoul, Korea.

No potential conflict of interest relevant to this article was reported.

Received: 27 May $2016 \bullet$ Revised: 15 Sep $2016 \bullet$ Accepted: 28 Sep 2016

pISSN: 2234-6163 • elSSN: 2234-6171 • https://doi.org/10.5999/aps.2016.43.6.523 • Arch Plast Surg 2016;43:523-528

\section{INTRODUCTION}

The use of acellular dermal matrix (ADM) in implant-based immediate breast reconstruction is increasing in popularity due to its proposed benefits [1], which include superior aesthetic outcomes, reduced capsular contracture, and reduced postoperative pain [2-4]. Since the pectoralis major muscle frequently provides inadequate inferolateral coverage for the implant, $\mathrm{ADM}$ can be a good substitute to allow complete subpectoral pocket creation.

The current ADMs available for breast reconstruction are offered as aseptic or sterile. "Aseptic" refers to the processing of tissue using methods to prevent, restrict, or minimize contamination with microorganisms from the environment, processing personnel, or equipment [5]. There is no sterility assurance level associated with aseptic processing. In contrast, "sterile" means 
that the tissue has completed a terminal sterilization process validated to a sterility assurance level of $10^{-6}$ [6].

AlloDerm (LifeCell Corp., Branchburg, NJ, USA), the first $\mathrm{ADM}$ used in breast reconstruction, is prepared by freeze-drying under aseptic conditions. It is the most recognized aseptic $\mathrm{ADM}$ by most surgeons, remains the most popular choice for postmastectomy breast reconstruction, and has been found to be very reliable and predictable [7]. MegaDerm (L\&C BIO, Seongnam, Korea), which has recently been introduced as a sterile and nonimmunogenic $\mathrm{ADM}$, is made using electronbeam sterilization to eliminate viruses, bacteria, and spores, achieving a $10^{-6}$ sterility level.

The use of a sterile ADM would theoretically be associated with a reduced risk of infection in breast reconstruction. Recently, several publications have described various correlations between the use of sterile ADM and postoperative complications in breast reconstruction [8-10]. However, no published studies have compared aseptic and sterile ADM in implantbased immediate breast reconstruction. The authors performed a retrospective study to evaluate whether sterile $\mathrm{ADM}$ offers a potential advantage over aseptic $\mathrm{ADM}$ with respect to postoperative complications, and specifically, rates of infection in implant-based immediate breast reconstruction. The authors have no vested interest of any kind in the materials or services referred to in this article.

\section{METHODS}

A retrospective review of the medical records was performed among patients who underwent implant-based immediate breast reconstruction with the application of aseptic ADM (Al-

\section{Fig. 1. ADM sling supporting the breast implant}

The superior border of the ADM was sutured to the inferior border of the pectoralis major with an interrupted 3-0 synthetic monofilament absorbable polyester. Total implant coverage was obtained. ADM, acellular dermal matrix.

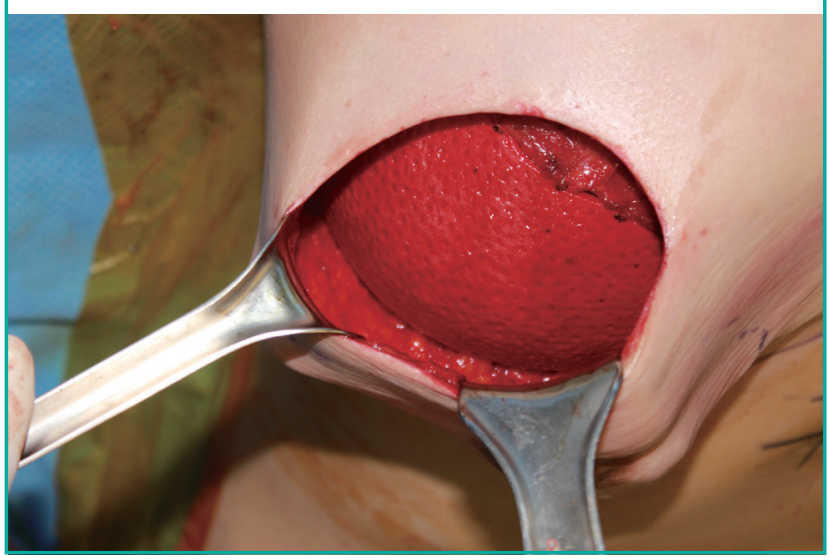

loDerm; size, $4 \times 12-4 \times 16 \mathrm{~cm}^{2}$; thickness, $2.31-3.30 \mathrm{~mm}$ ) or sterile ADM (MegaDerm; size, $4 \times 12-6 \times 16 \mathrm{~cm}^{2}$; thickness, $1.5-2.3 \mathrm{~mm}$ ) in our institution. Exclusion criteria included the following: (1) patients whose breast reconstructions were performed in a delayed fashion or involved either tissue expander placement or autologous reconstruction; (2) patients who received radiation therapy at any time; (3) patients with significant comorbidities that could affect wound healing; (4) breast reconstructions performed before April 2013, the first use of sterile ADM in our institution; and (5) patients who had not yet completed a 6-month follow-up visit.

General information on patient demographics, clinical course, and postoperative complications was collated. Patients were allocated to either the aseptic ADM group (AlloDerm) or the sterile ADM group (MegaDerm).

All breast reconstructions were performed according to the same surgical protocol by a single surgeon (J.H.L.). After completion of skin-sparing mastectomy, a subpectoral dissection was performed and the inferior and inferomedial attachments of the pectoralis major were released. An implant was inserted into a newly created submuscular pocket. The pectoralis muscle was used to cover the implant superiorly. An ADM sling was used to cover the implant inferolaterally. The $\mathrm{ADM}$ was sutured with 3-0 synthetic monofilament absorbable polyester (BIOSYN, Covidien, Mansfield, MA, USA) to the inframammary fold along the entire lower border of the breast pocket with interrupted sutures. Laterally, the $\mathrm{ADM}$ was sutured to the serratus fascia to define the lateral boundary of the breast pocket along the anterior axillary line (Fig. 1).

Postoperatively, two closed-suction drains were placed into the supra-ADM plane (between the $\mathrm{ADM}$ and the skin) and the

Fig. 2. Infection after implant-based immediate breast reconstruction

Intravenous antibiotic treatment or implant removal was required for infectious complications in implant-based immediate breast reconstructions

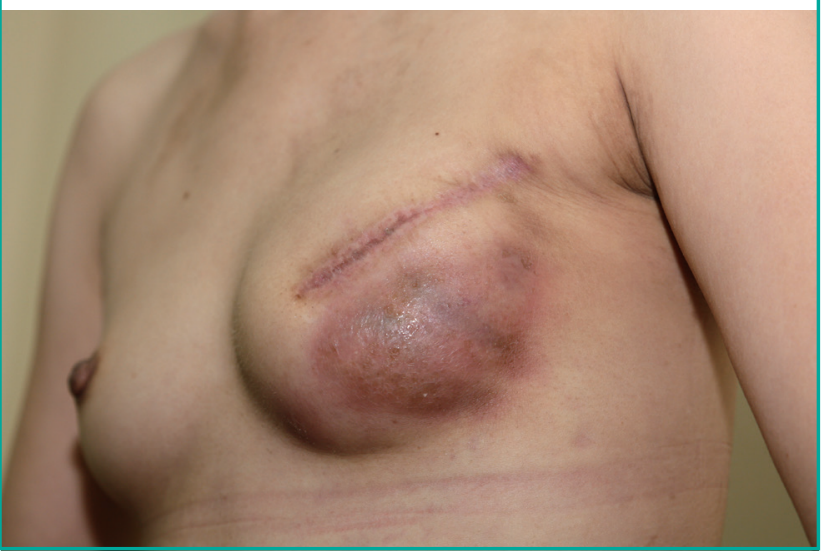


sub-ADM plane (between the implant and the $\mathrm{ADM}$ ), until output decreased to $<20 \mathrm{~mL}$ over 24 hours. Postoperative antibiotics were continued until the drains were removed. Complications were analyzed, including infection, flap necrosis, capsular contracture, seroma, and hematoma. Infection was defined as erythematous changes on the affected breast with general symptoms of infection, including a sensation of heat on the breast, a generalized febrile sense, and fever (Fig. 2). Infectious complications were divided into 2 types: (1) infections requiring hospital readmission and intravenous antibiotics and (2) infections requiring surgery, including implant change or explantation. Any infectious complications encountered during the follow-up period were regarded as significant. Cultured microorganism data were collected in cases of infection requiring surgery. Flap necrosis was defined as skin necrosis only along the incision, without any erythematous change in the breast (Fig. 3). Seroma was defined as fluid collection on the affected breast that required ultrasonogram-guided aspiration after the removal of both drains. Overall explantation rates for any cause were also analyzed.

The Fisher's exact test and chi-square test were performed to compare the outcomes between the aseptic $\mathrm{ADM}$ group and the sterile $\mathrm{ADM}$ group. P-values $<0.05$ were considered to indicate statistical significance.

\section{RESULTS}

Eighty-eight breast reconstructions from April 2013 to January 2016 were included in this study. All 88 patients underwent unilateral implant-based immediate breast reconstruction using a textured silicone gel implant (Allergan Corp., Irvine, CA, USA).

Fig. 3. Flap necrosis after implant-based immediate breast reconstruction

An additional operation was necessary to treat cases of flap necrosis that occurred along the incision after implant-based immediate breast reconstructions with acellular dermal matrix.

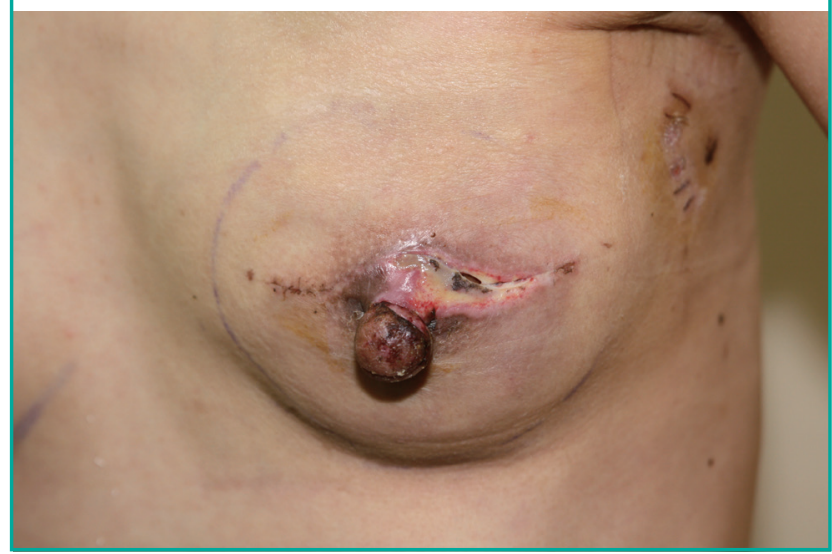

Twenty patients were reconstructed with aseptic $\mathrm{ADM}$ and 68 patients with sterile $\mathrm{ADM}$. The mean follow-up periods were 14 months in the aseptic ADM group and 12 months in the sterile ADM group.

Patients in the 2 groups had similar characteristics in terms of age, body mass index, medical comorbidities, implant size, breast cancer stage, and the need for adjuvant antineoplastic treatment (Table 1).

Infectious complications were encountered in 3 patients (15.0\%) from the aseptic $\mathrm{ADM}$ group, including 1 case of infection requiring conservative treatment with intravenous antibiotics (5.0\%) and 2 cases of infection requiring surgery (10.0\%). In the sterile $\mathrm{ADM}$ group, the overall incidence of infectious complications was 7 (10.3\%), including 2 cases of infection requiring IV antibiotics (2.9\%) and 5 cases of infection requiring surgery (7.4\%). There were fewer infectious complications in the sterile ADM group, but the difference failed to reach statistical significance. Flap necrosis was encountered in 1 case (5.0\%) in

\section{Table 1. Characteristics of the breast reconstruction} patients

\begin{tabular}{|c|c|c|c|}
\hline Characteristic & $\begin{array}{l}\text { AlloDerm } \\
(n=20)\end{array}$ & $\begin{array}{l}\text { MegaDerm } \\
(\mathrm{n}=68)\end{array}$ & P-value \\
\hline \multicolumn{4}{|l|}{ Age } \\
\hline Mean & 46.1 & 45.8 & 0.568 \\
\hline Range & $32-58$ & $31-62$ & \\
\hline \multicolumn{4}{|l|}{ Body mass index $\left(\mathrm{kg} / \mathrm{m}^{2}\right)$} \\
\hline Mean & 22.3 & 23.1 & 0.529 \\
\hline Range & $17.5-32.3$ & $17.7-32.1$ & \\
\hline \multicolumn{4}{|l|}{ Comorbid conditions } \\
\hline Smoking & 0 & 0 & - \\
\hline Diabetes & 0 & 0 & - \\
\hline Obese ${ }^{\text {a) }}$ & $2(10.0)$ & $7(10.3)$ & 0.814 \\
\hline Specimen weight, mean \pm SD (g) & $349.6 \pm 158.2$ & $358.1 \pm 170.1$ & 0.795 \\
\hline \multicolumn{4}{|l|}{ Implant size $(\mathrm{mL})$} \\
\hline$<150$ & $3(15.0)$ & $9(13.2)$ & 0.783 \\
\hline $150-250$ & $11(55.0)$ & $39(57.4)$ & 0.711 \\
\hline $250-350$ & $4(20.0)$ & $13(19.1)$ & 0.802 \\
\hline$>350$ & $2(10.0)$ & $7(10.3)$ & 0.357 \\
\hline \multicolumn{4}{|l|}{ Breast cancer stage } \\
\hline 0 & $7(35.0)$ & $21(30.9)$ & 0.746 \\
\hline 1 & $8(40.0)$ & $28(41.2)$ & 0.692 \\
\hline 2 & $3(15.0)$ & $12(17.6)$ & 0.688 \\
\hline 3 & $2(10.0)$ & $7(10.3)$ & 0.412 \\
\hline 4 & 0 & 0 & - \\
\hline Adjuvant CTx & $7(35.0)$ & $20(29.4)$ & 0.529 \\
\hline Neoadjuvant CTx & $1(5.0)$ & $5(7.4)$ & 0.716 \\
\hline ALND & $7(35.0)$ & $22(32.4)$ & 0.789 \\
\hline NAS & $11(55.0)$ & $40(58.9)$ & 0.39 \\
\hline Bilateral reconstruction & 0 & 0 & - \\
\hline
\end{tabular}


Table 2. Complications of breast reconstruction patients

\begin{tabular}{|llcc|}
\hline Variable & $\begin{array}{c}\text { AlloDerm } \\
(\mathbf{n}=\mathbf{2 0})\end{array}$ & $\begin{array}{c}\text { MegaDerm } \\
(\mathbf{n}=\mathbf{6 8})\end{array}$ & P-value \\
\hline Infection & $3(15.0)$ & $7(10.3)$ & 0.144 \\
$\quad$ Infection requiring IV antibiotics & $1(5.0)$ & $2(2.9)$ & 0.283 \\
$\quad$ Infection requiring surgery & $2(10.0)$ & $5(7.4)$ & 0.407 \\
Flap necrosis & $1(5.0)$ & $5(7.4)$ & 0.182 \\
Capsular contracture & $4(20.0)$ & $10(14.7)$ & 0.498 \\
Baker grade I, II & $3(15.0)$ & $7(10.3)$ & 0.144 \\
Baker grade III, IV & $1(5.0)$ & $3(4.4)$ & 0.605 \\
Seroma & $2(10.0)$ & $10(14.7)$ & 0.163 \\
Hematoma & 0 & $1(1.5)$ & 0.823 \\
Explantation by any cause & $2(10.0)$ & $6(8.8)$ & 0.235 \\
\hline Values are presented as number (\%). & & \\
\hline
\end{tabular}

the aseptic $\mathrm{ADM}$ group, and in 5 cases (7.4\%) in the sterile $\mathrm{ADM}$ group. The incidence of Baker grade I or II capsular contracture was $3(15.0 \%)$ in the aseptic ADM group and 7 (10.3\%) in the sterile ADM group. The incidence of Baker grade III or IV was $1(5.0 \%)$ in the aseptic ADM group and 3 $(4.4 \%)$ in the sterile ADM group. Seroma was encountered in 2 breasts $(10.0 \%)$ in the aseptic $\mathrm{ADM}$ group, and 10 breasts (14.7\%) in the sterile $\mathrm{ADM}$ group. The sterile $\mathrm{ADM}$ group showed a higher rate of seroma than the aseptic ADM group; however, this discrepancy was not statistically significant. Overall, the explantation rate and incidence of hematomas were not significantly different between the 2 groups (Table 2 ). Cultured microorganisms in infectious cases from the aseptic ADM group were identified as methicillin-susceptible Staphylococcus aureus (MSSA) in 1 case and methicillin-resistant Staphylococcus aureus (MRSA) in 1 case. In the 5 infectious complication cases in the sterile ADM group, we found 2 cases of MSSA, 2 cases of MRSA, and 1 case of Serratia marcescens (Table 3 ).

\section{DISCUSSION}

$\mathrm{ADM}$ is a structurally intact tissue matrix that can act as a biological scaffold to induce tissue ingrowth, angiogenesis, and tissue regeneration. Since Breuing and Warren [11] reported a successful outcome of breast reconstruction with ADM in 2005, the use of $\mathrm{ADM}$ with breast reconstruction has been a well-accepted surgical option. After skin-sparing mastectomy, ADM can be applied to form inferolateral coverage of the pocket of the tissue expander (two-stage breast reconstruction) or the pocket of the implant (direct-to-implant immediate breast reconstruction).

AlloDerm, the first $\mathrm{ADM}$ used in breast reconstruction, has become the most recognized aseptic ADM worldwide. Aseptic $\mathrm{ADM}$ undergoes an aseptic process, including cryopreservation,
Table 3. Identified microorganisms in infectious complication cases requiring surgery

\begin{tabular}{|lcc|}
\hline Variable & AlloDerm $(\mathbf{n = 2})$ & MegaDerm $(\mathbf{n = 5})$ \\
\hline MSSA & 1 & 2 \\
MRSA & 1 & 2 \\
Serratia marcescens & - & 1 \\
\hline MSSA, methicillin-susceptible Staphylococcus aureus; MRSA, methicillin-resistant \\
Staphylococcus aureus.
\end{tabular}

and is freeze-dried. As the benefits of using AlloDerm in implant-based breast reconstruction have become obvious, AlloDerm remains one of the most popular, reliable, and predictable choices for postmastectomy breast reconstruction.

Despite the removal during manufacture of all the cellular components in ADM that could cause rejection and inflammation, several reports have suggested that the use of ADM may be associated with elevated rates of infection and seromas $[12,13]$. Chun et al. [2] reported a series of 415 immediate breast reconstructions performed on 283 patients. The authors found that both seroma and infection rates were higher in the aseptic ADM group than in the non-ADM group. Similarly, Liu et al. [12] also found in their large series of 470 immediate reconstructions that the infection rate in the ADM group was significantly higher than in the non-ADM group. Antony et al. [13] reported a higher incidence of overall complications, specifically, seroma and reconstructive failures, in their $\mathrm{ADM}$ group versus their non$\mathrm{ADM}$ group and their previously published non-ADM group. Similarly, Lanier et al. [14] found a significantly higher rate of infection, reoperation, explantation, and overall complications when they compared their aseptic ADM group to their nonADM group.

In contrast, several studies have reported that $\mathrm{ADM}$ was not associated with an elevated rate of infectious complications. In his case series, Nahabedian [15] showed that the overall incidence of infection was essentially the same in the ADM and non-ADM groups. Vardanian et al. [16] also found that their aseptic $\mathrm{ADM}$ group had a similar safety profile to their nonADM group.

In terms of infectious complication for the use of $\mathrm{ADM}$ in breast reconstruction, the use of sterile $\mathrm{ADM}$ would theoretically be associated with a reduced risk of infection in breast reconstruction. "Sterile" means that the ADM has completed a terminal sterilization process validated to a sterility assurance level of 10-6. In other words, a microorganism has a one in 1 million chance of surviving the sterilization process.

Buseman et al. [8] reported a comparative study of aseptic and sterile ADM. The authors found that sterile ADM has a risk of infection equivalent to that of aseptic $\mathrm{ADM}$. However, the ster- 
ile $\mathrm{ADM}$ group showed a higher incidence of seroma than the aseptic ADM group. Yuen et al. [17] reported a significantly higher incidence of infection and seroma in the sterile ADM group than in the aseptic ADM group. Previous studies comparing aseptic and sterile $\mathrm{ADM}$ have been performed with readyto-use AlloDerm as the sterile ADM. MegaDerm, which has been recently introduced as a sterile ADM in South Korea, is prepared using an electron-beam sterilization process. No reports have been published on the use of MegaDerm for breast reconstruction.

Our results showed no statistically significant differences between the 2 groups in terms of infection, flap necrosis, capsular contracture, seroma, hematoma, or overall explantation rates. It should be noted that sterile $\mathrm{ADM}$ showed complication rates equivalent to those observed using aseptic $\mathrm{ADM}$, including rates of infectious complications. However, our study has several limitations, including its retrospective nature. In practice, many potential factors could cause infectious complications in implant-based immediate breast reconstruction. To minimize the effect of all other factors except ADM, all cases before the first use of sterile ADM (MegaDerm) at our institution were excluded, and the only 20 cases were included in the aseptic group. The number of cases in the aseptic group was smaller than in recent studies of outcomes of ADM in breast reconstruction. In our study, no cases of red breast syndrome occurred. Since red breast syndrome also appears as erythema along the inferior pole of the breast, it is possible to misdiagnose a case as infection. Despite trying to render all conditions between the 2 groups as similar as possible, some factors that could influence the outcomes remained. Because the ADM was produced in a rectangular shape, trimming or removal of unnecessary parts of the $\mathrm{ADM}$ was performed to fit the inferolateral breast pocket. The amounts of microorganisms that could cause infectious complications were therefore different within the same group. Three breast surgeons performed skin-sparing mastectomies at our institution over the course of this study. A breast surgeon who leaves behind very well-vascularized mastectomy flaps affords the reconstructive surgeon an optimal setting to minimize complications. For example, the thickness of the skin after a skin-sparing mastectomy is important, because this skin flap can cause flap necrosis or infection. Additionally, the operating time may differ among surgeons, with longer operating time being a factor associated with infection. To minimize the influence of these biases on the outcomes of implant-based immediate breast reconstruction, a randomized controlled prospective trial would be necessary.

To summarize, sterile ADM did not provide improved results related to infectious complications, contrary to the theoretical expectation. In other words, the sterilization of $\mathrm{ADM}$ for the sole purpose of minimizing infectious complications was unnecessary. It is noteworthy that infectious complications in implant-based immediate breast reconstructions can be influenced more by contamination during the intraoperative procedure or postoperative care than by contamination of the $\mathrm{ADM}$ product itself. Although the sterilization of $\mathrm{ADM}$ did not demonstrate more preferable outcomes in terms of infection, sterile $\mathrm{ADM}$ has some advantages, including resistance to degradation by collagenase, improvement of durability, and strength due to crosslinking [18]. These biomechanical characteristics of sterile $\mathrm{ADM}$ need to be investigated in further studies.

\section{REFERENCES}

1. Salzberg CA. Direct-to-implant breast reconstruction. Clin Plast Surg 2012;39:119-26.

2. Chun YS, Verma K, Rosen H, et al. Implant-based breast reconstruction using acellular dermal matrix and the risk of postoperative complications. Plast Reconstr Surg 2010;125: 429-36.

3. JoAnna Nguyen T, Carey JN, Wong AK. Use of human acellular dermal matrix in implant- based breast reconstruction: evaluating the evidence. J Plast Reconstr Aesthet Surg 2011; 64:1553-61.

4. Spear SL, Pelletiere CV, Lockwood M. Immediate breast reconstruction with tissue expanders and AlloDerm. In: Spear SL, Willey SC, Robb GL, et al., editors. Surgery of the breast: principles and art. 2nd ed. Philadelphia: Lippincott Williams \& Wilkins; 2006. p.484-8.

5. United States Pharmacopeial Convention. 1211: Sterility testing. United States Pharmacopeia and National Formulary (USP25-NF20). Rockville: United States Pharmacopeial Convention; 2002.

6. Centers for Disease Control and Prevention (CDC). Update: allograft-associated bacterial infections: United States, 2002. MMWR Morb Mortal Wkly Rep 2002;51:207-10.

7. Losken A. Early results using sterilized acellular human dermis (Neoform) in post-mastectomy tissue expander breast reconstruction. Plast Reconstr Surg 2009; 123:1654-8.

8. Buseman J, Wong L, Kemper P, et al. Comparison of sterile versus nonsterile acellular dermal matrices for breast reconstruction. Ann Plast Surg 2013;70:497-9.

9. Weichman KE, Wilson SC, Saadeh PB, et al. Sterile "readyto-use" AlloDerm decreases postoperative infectious complications in patients undergoing immediate implant-based breast reconstruction with acellular dermal matrix. Plast Reconstr Surg 2013;132:725-36. 
10. Venturi ML, Mesbahi AN, Boehmler JH 4th, et al. Evaluating sterile human acellular dermal matrix in immediate expander-based breast reconstruction: a multicenter, prospective, cohort study. Plast Reconstr Surg 2013;131:9e-18e.

11. Breuing KH, Warren SM. Immediate bilateral breast reconstruction with implants and inferolateral AlloDerm slings. Ann Plast Surg 2005;55:232-9.

12. Liu AS, Kao HK, Reish RG, et al. Postoperative complications in prosthesis-based breast reconstruction using acellular dermal matrix. Plast Reconstr Surg 2011;127:1755-62.

13. Antony AK, McCarthy CM, Cordeiro PG, et al. Acellular human dermis implantation in 153 immediate two-stage tissue expander breast reconstructions: determining the incidence and significant predictors of complications. Plast Reconstr Surg 2010;125:1606-14.

14. Lanier ST, Wang ED, Chen JJ, et al. The effect of acellular dermal matrix use on complication rates in tissue expander/ implant breast reconstruction. Ann Plast Surg 2010;64:6748.

15. Nahabedian MY. AlloDerm performance in the setting of prosthetic breast surgery, infection, and irradiation. Plast Reconstr Surg 2009;124:1743-53.

16. Vardanian AJ, Clayton JL, Roostaeian J, et al. Comparison of implant-based immediate breast reconstruction with and without acellular dermal matrix. Plast Reconstr Surg 2011; 128:403e-410e.

17. Yuen JC, Yue CJ, Erickson SW, et al. Comparison between freeze-dried and ready-to-use AlloDerm in alloplastic breast reconstruction. Plast Reconstr Surg Glob Open 2014;2: e119.

18. Lee JH, Kim HG, Lee WJ. Characterization and tissue incorporation of cross-linked human acellular dermal matrix. Biomaterials 2015;44:195-205. 